\title{
Dual-Resonance Long Period Grating in Fiber Loop Mirror Based Platform for Cheap Biomedical Sample Detection with High Resolution
}

\author{
Renata Zawisza*1 $^{* 1}$, Leszek R Jaroszewicz ${ }^{1}$, Anna Celebańska ${ }^{2}$, Predrag Mikulic ${ }^{2}$ and Wojtek J Bock ${ }^{2}$ \\ ${ }^{1}$ Institute of Applied Physics, Military University of Technology, Poland \\ ${ }^{2}$ Photonics Research Center, Université du Québec en Outaouais, Canada
}

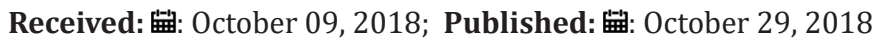

*Corresponding author: Renata Zawisza, Institute of Applied Physics, Military University of Technology, Poland

\begin{abstract}
A dual-resonance long period grating (DR-LPG) embedded in fiber loop mirror (FLM) was employed as a sample for the development of the biosensor application. The DR-LPG) acts as a sensing part for biological molecules detection, whereas FLM enhances the rapid signal monitoring, which oversees the wavelength filtering properties. Herein, the DR-LPG surface was functionalized with biotin in terms of the avidin binding reaction detection. The detection mechanism is based on the alteration the refractive index of the DR-LPG surrounding monitored by the tracking interference dips changing. Moreover, by applying a broadband diode and photodetector we ensured the low-cost of the system.
\end{abstract}

Keywords: Dual- Resonance Long Period Grating (DR-LPG); Fiber Loop Mirror (FLM); Biotin- Avidin Reaction; Biosensing Platform.

Abbreviations: DR-LPG: Dual- Resonance Long Period Grating; FLM: Fiber Loop Mirror; LPG: Long Period Grating; RI: Refractive Index; APTES: Amino Propyl Tri Ethoxy Silane; EDC: 1-Ethyl-3-(3-Dimethylaminopropyl)Carbodiimide; PBS: Phosphate Buffer Saline; LOF: Lab-On-a-Fiber

\section{Introduction}

An optical fiber based sensor are very useful in many applications because of the compact size, electrical interference independence and suitability for remote sensing [1]. Recently, a significant effort has been devoted for the long period grating (LPG) - based sensors for medical application [2,3]. The real-time detection, high sensitivity and accuracy are desire properties for the medical sensors in terms of the facilitate prompt disease diagnosis [3]. In spite of the high sensitivity of the LPG, the main asset is possibilities of modifying the optical structure with thin functional coatings in terms of facilitating highly selective and fast measurement in real time with the reported resolution of refractive index (RI) change up to 10-6 [4]. To detect any molecular elements, the LPG surface needs to be functionalized by e. g. enzymes [5], gold nanoparticles [6], antibodies [7] or cross- linking bioreceptors [8]. The LPG is an optical periodic structure, where transmission spectrum consists of the resonance dips at discrete wavelength [9]. When it comes to achieve very sensitive structure, the LPG should work close to the turning point, where the dual-resonance appears.

Such dual- resonance long period grating (DR-LPG) has been shown to be ultra-sensitive [10] regarding the micro-scale labelfree detection [11]. The detailed theory of the DR-LPG structure can be found in $[12,13]$. To satisfy the need for very accuracy molecules detection and to maintain a stable temperature of the measurement, the DR-LPG can be combining with other optical elements [14]. Among various solution, the fiber loop mirror (FLM) provides several advantages, including high extinction ratio, and low cost of final arrangement. The part of high birefringence fiber (Hi-Bi fiber) from FLM structure ensures ambient temperature changes controlling by the interference dips shifting/amplitude intensity changing observation. Moreover due to filtering properties of the FLM, it provides possibilities of control of the wavelength spacing between interference dips, limitation of the temperature and strain cross- sensitivities and consequently enhancement of the sensitivity of the specific sample detection [15].In this paper, for the first time of our knowledge, we present the combined structure of the DR-LPG in the FLM for biotin-avidin reaction as a potential platform for medical application.

In particular, the described optical platform can be applied as a sensor for limitless biomarkers or molecules, similarly to LPG which was already used to detect, e.g. bacteria [16]. It is possible due to the ultra-high sensitivity towards a refractive index of surrounding environment. Moreover, the introduction of a reception layer to 
the sensing system ensures its selectivity exclusively towards the desired analyte.

Here, we present a proof of concept of the application of our sensing platform by applying the well-known biological model, biotin-avidin complex. Esmond Snell discovered the biotinavidin interaction during the investigation on "egg-white injury" disorder in 1941 [17]. Biotin calls also vitamin H, it is an crucial compound taking part in the metabolism of fatty acids and amino acids. The protein, avidin from raw egg white has capability to bind biotin causing its deficiency in mammalian bodies. The avidinbiotin binding belongs to the most robust noncovalent biological interaction with a dissociation constant 10-15 [18]. In the presented system, biotin is applied as a receptor, immobilized covalently to the surface, able to selectively capture of avidin dissolved in the solution.

\section{Methods \\ The Experimental Set-Up}

The DR-LPG was produced using amplitude mask technique, where the part of bare fiber was exposed on UV-radiation using $\mathrm{KrF}$ Excimer laser (Lumonics ${ }^{\mathrm{TM}}$ Lasers: Pulse Master ${ }^{\circledR}-840$ ) emitting at $248 \mathrm{~nm}$. The period of the used DR-LPG was $217 \mu \mathrm{m}$. The experimental platform used for monitoring biotin-avidin reaction is shown in Figure 1a and has been in details described in [19]. The transmission spectra of proposed platform are shown in Figure 1b, where the DR-LPG in FLM spectrum was adjust by a polarization controller in terms of maximum set-up response.

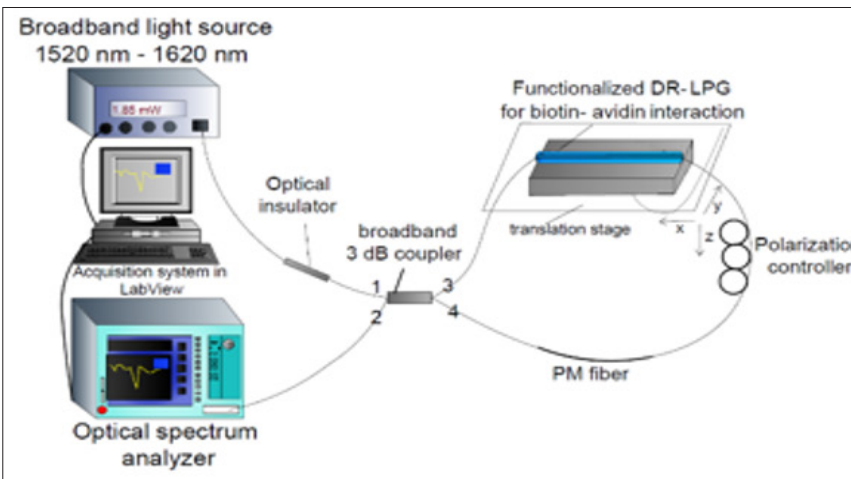

A

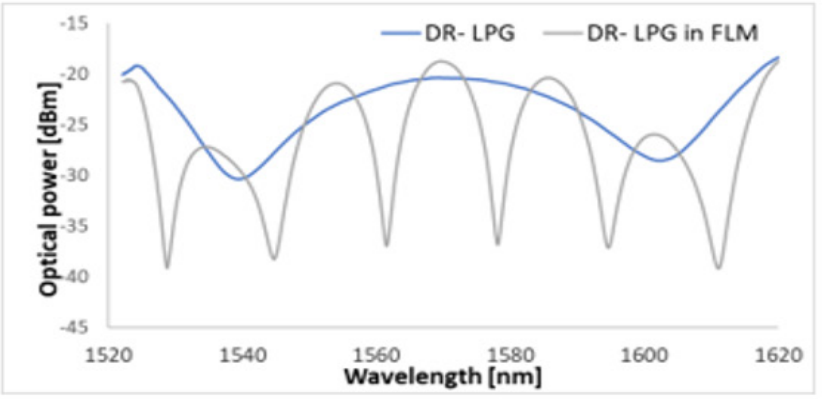

B

Figure 1: The scheme of the experimental platform for biotin-avidin interaction (a) and transmission spectra of DR-LPG (blue line) and DR-LPG in FLM (grey line) (b).

\section{Receptor Immobilization Procedure}

The DR-LPG surface was cleaned from organic impurities by soaking in hydrochloric acid (aq.)/methanol (1:1, v/v) mixture and next in concentrated sulfuric acid for $30 \mathrm{~min}$, followed by plentiful rinsing with water. After that, the platform was dried in vacuum for 15 min and transferred for the aminization process. The DR-LPG was locked into the desiccator chamber together with trays of $30 \mu \mathrm{l}$ of 3-aminopropyltriethoxysilane (APTES) and $10 \mu \mathrm{l}$ of triethylamine for $30 \mathrm{~min}$ and/or $2 \mathrm{~h}$ in the argon atmosphere [20]. In the next step, the biotins carboxylic group was activated by EDC/NHS to form an amide bond with amine groups of the APTES covered surface. For this, the biotin powder was dissolved in the mixture of water and dimethylformamide $(1: 1, \mathrm{v} / \mathrm{v})$ containing $0.8 \mathrm{M}$ 1-ethyl-3(3-dimethylaminopropyl)carbodiimide hydrochloride (EDC) and $60 \mathrm{mM}$ N-hydroxysuccinimide (NHS). The final concentration of biotin was equal $1 \mathrm{mg}$ ml-1. Next, the APTES covered platform was immersed in the prepared solution for 1 hour in a sealed chamber. After this time, DR-LPG was rinsed with water and exposed to avidin for $30 \mathrm{~min}$. Avidin solution was prepared by it dissolving in phosphate buffer saline (PBS) $\mathrm{pH}=7.4$ to obtain concentration equal $1 \mathrm{mg} \mathrm{ml}-1$. Figure 2 shows the scheme of functionalised DRLPG surface with a biotin-avidin interaction.

\section{Receptor Immobilization Procedure}

The DR-LPG surface was cleaned from organic impurities by soaking in hydrochloric acid (aq.)/methanol (1:1, v/v) mixture and next in concentrated sulfuric acid for $30 \mathrm{~min}$, followed by plentiful rinsing with water. After that, the platform was dried in vacuum for $15 \mathrm{~min}$ and transferred for the aminization process. The DR-LPG was locked into the desiccator chamber together with trays of $30 \mu \mathrm{l}$ of 3-aminopropyltriethoxysilane (APTES) and $10 \mu$ l of triethylamine for $30 \mathrm{~min}$ and/or $2 \mathrm{~h}$ in the argon atmosphere [20]. In the next step, the biotins carboxylic group was activated by EDC/NHS to form an amide bond with amine groups of the APTES covered surface. For this, the biotin powder was dissolved in the mixture of water and dimethylformamide $(1: 1, \mathrm{v} / \mathrm{v})$ containing $0.8 \mathrm{M}$ 1-ethyl-3(3-dimethylaminopropyl)carbodiimide hydrochloride (EDC) and $60 \mathrm{mM}$ N-hydroxysuccinimide (NHS). The final concentration of biotin was equal $1 \mathrm{mg} \mathrm{ml}^{-1}$. Next, the APTES covered platform was immersed in the prepared solution for 1 hour in a sealed chamber. After this time, DR-LPG was rinsed with water and exposed to avidin for $30 \mathrm{~min}$. Avidin solution was prepared by it dissolving in phosphate buffer saline (PBS) $\mathrm{pH}=7.4$ to obtain concentration equal $1 \mathrm{mg} \mathrm{ml}^{-1}$. Figure 2 shows the scheme of functionalised DRLPG surface with a biotin-avidin interaction. 


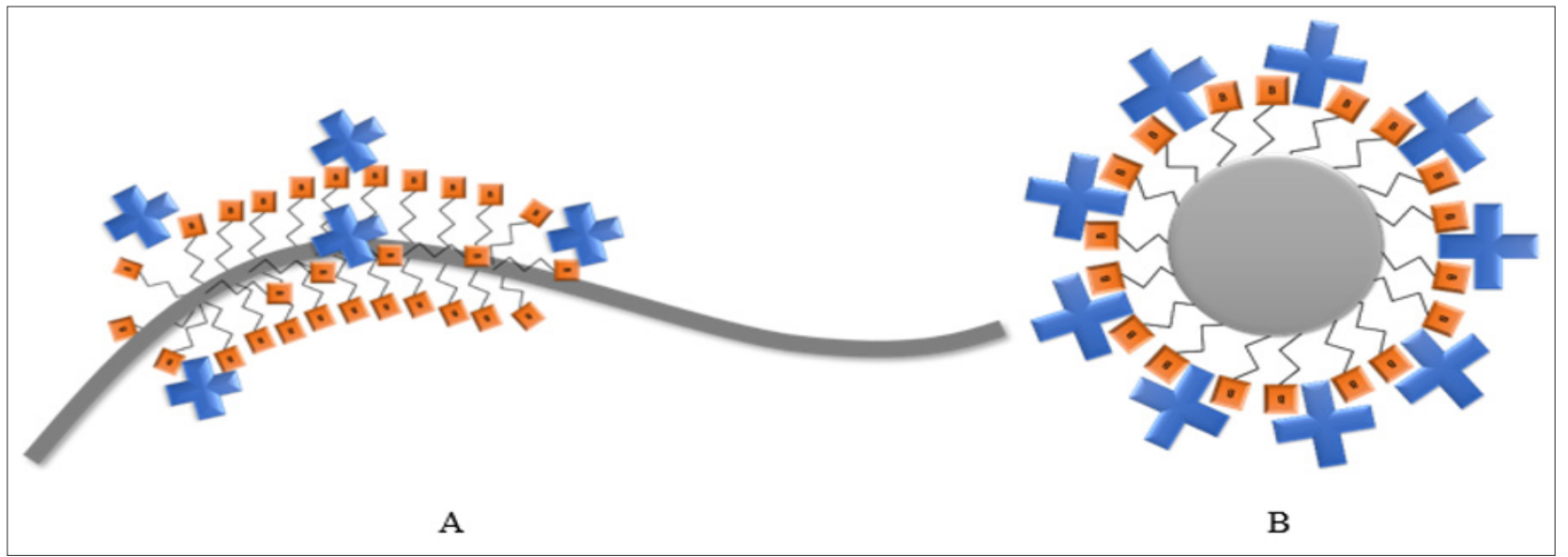

Figure 2: The scheme of the DR-LPG functionalised with a biotin (orange square) interacting with avidin (blue cross) from the solution: a) the whole platform, b) the cross-section of the platform.

\section{Results}

All presented measurement data was performed in a fresh PBS imitating human blood serum, and every step of functionalization process was followed by the DR-LPG excessive wash to remove weakly bonded biomolecules. This procedure allowed to obtain a reliable response. Moreover, to maintain stable physical conditions the temperature was monitored, and the DR-LPG was kept in a constant volume of PBS. The introduction of the sensor into a narrow tube allowed to control and suppressed unwanted solution evaporation. The spectrum was recorded three times in PBS. Firstly, after the surface sensor silanization process (Figure 3), light blue line). Secondly, after the biotin covalently attachment to the sensor (dark blue line). And thirdly, afterword the sensor interaction with avidin (grey line). The red dashed lines in Figure 3 mark the notches of the DR-LPG which corresponds with those from Figure $1 \mathrm{~b}$ and only these minima are considered in regards of sensing properties of the device. When the ambient refractive index varying, the DRLPG notches (around $1530 \mathrm{~nm}$ and $1615 \mathrm{~nm}$ ) shifting toward each other, and hence, the amplitude of the interference dips of the DRLPG in FLM is changed.

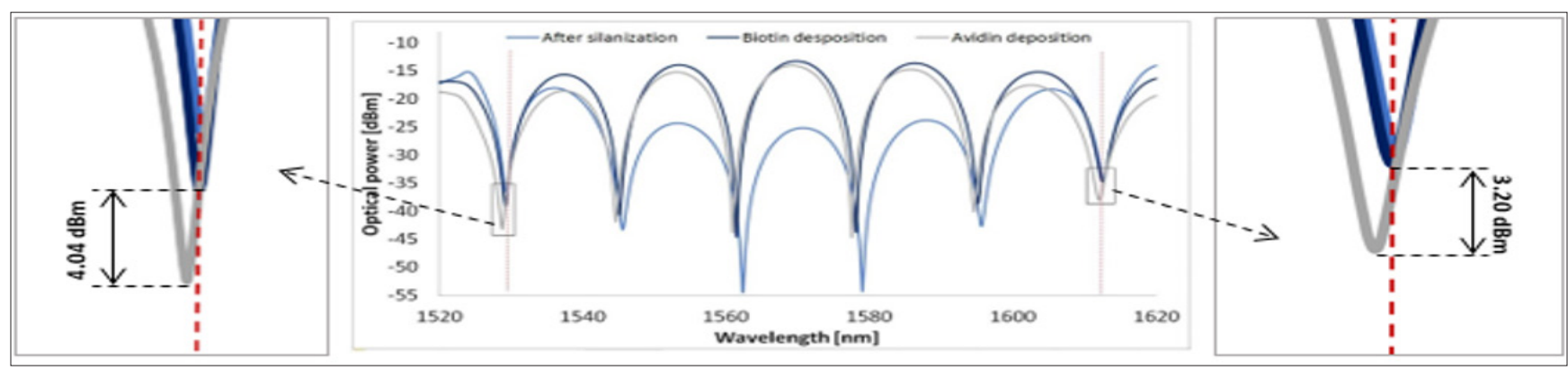

Figure 3: The experimental platform response for DR-LPG surface silanization (light blue line), DR- LPG immersed on PBS after biotin deposition (dark blue line) and DR- LPG immersed n PBS after avidin deposition (grey line). The left/right magnifications show $1530 \mathrm{~nm}$ and $1615 \mathrm{~nm}$ region, respectively.

The fact, that amplitude of the transmission spectrum of the DR-LPG in FLM significantly increase after biotin disposition means that biotin was stable coated to the DR- LPG surface and BSA could not rinse it from the surface. As can be seen from Figure 3, only the amplitude of the covered by the DR-LPG notches interference dips decrease after avidin attachment. The observed change is estimated to $4.04 \mathrm{dBm}$ and $3.20 \mathrm{dBm}$, respectively to $1530 \mathrm{~nm}$ and $1615 \mathrm{~nm}$. The experiment confirmed that presented platform combined with appropriate receptor can be applied as a sensor for biological analytes detection.

\section{Conclusion}

We presented a potential application of the DR-LPG in FLM platform for label-free biochemical detection. The proposed solution fits squarely into technology named Lab-On-a-Fiber (LOF) (more specifically Lab-around-Fiber), which is dedicated to devices focused on the design and development of advanced fiber optic nanoprobes for biological applications. Hence, the LOF enables continuous monitor of detected samples. Adapting FLM in the sensor probe (DR-LPG) influences on avoiding thermal and strain cross- 
sensitives, and this limitation dealing with pure unconventional light matter interaction in confined small-scale volumes. Despite of many asset of the FLM configuration, it ensures relatively high sensitivity due to the phase shift keying. Finally, throughout adoption of the laser diode, (broadband light source) photodetector and the commercially available optical passive elements with the telecommunication standards, the DR-LPG in the FLM configuration provides the low- cost usable sensor implementation. Therefore, we can conclude that proposed platform can monitor the biotinavidin interaction and is good candidate to some other biological sample detection as a medical application.

\section{Acknowledgment}

This research was funded by the financial support of the Natural Sciences and Engineering Research Council of Canada for the SPI/NSERC Industrial Research Chair in Photonic Sensing Systems for Safety and Security Monitoring. The investigation was also supported by the internal MUT project No. RMN 08/690 as well as project under the Ministry of National Defense Republic of Poland Program-Research Grant MUT project No. 13-995.

\section{References}

1. Brogan KL, Walt DR (2005) Optical fiber-based sensors: application to chemical biology. Current Opinion in Chemical Biology 9(5): 494-500.

2. Petrović MD, Petrovic J, Daničić A, Vukčević M, Bojović B, et al. (2014) Non-invasive respiratory monitoring using long-period fiber grating sensors. Biomedical Optics Express 5(4): 1136-1144.

3. Marques L, Hernandez FU, James SW, Morgan SP, Clark M, et al. (2016) Highly sensitive optical fibre long period grating biosensor anchored with silica core gold shell nanoparticles. Biosensors and Bioelectronics 75: 222-231.

4. Korposh S, James S, Tatam R, Lee SW (2012) Optical fibre long-period gratings functionalised with nanoassembled thin films: approaches to chemical sensing Cuadrado-Laborde (Ed.) Current Trends in Short-and Long-Period Gratings InTech, Croatia pp. 237-2264.

5. Badmos AA, Sun Q, Sun Z, Zhang J, Yan Z, et al. (2017) Enzymefunctionalized thin-cladding long-period fiber grating in transition mode at dispersion turning point for sugar-level and glucose detection. Journal of Biomedical Optics 22(2): 27003.

6. Heidemann BR, Chiamenti I, Oliveira MM, Muller M, Fabris JL, et al. (2018) Functionalized Long Period Grating- Plasmonic Fiber Sensor Applied to the Detection of Glyphosate in Water. Journal of Lightwave Technology 36(4): 50.

7. Liang L, Leonel L, Ricardo M, Stephen C, Morgan SP, et al. (2018) Highly sensitive label-free antibody detection using a long period fibre grating sensor. Sensors and Actuators B: Chemical 271(15): 24-32.

\section{ISSN: 2574-1241}

DOI: $10.26717 /$ BJSTR.2018.10.001967

Renata Zawisza. Biomed J Sci \& Tech Res

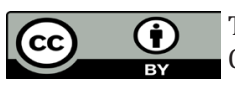

This work is licensed under Creative

Commons Attribution 4.0 License

Submission Link: https://biomedres.us/submit-manuscript.php
8. Chen X, Liu Ch, Hughes MD, Nagel DA, Hine AV, et al. (2015) EDCMediated Oligonucleotide Immobilization on a Long Period Grating Optical Biosensor. Journal of Biosensors \& Bioelectronics 6(2): 173.

9. Keith J, Puckett S, Pacey GE (2003) Investigation of the fundamental behavior of long-period grating sensors. Talanta 61(4): 417-421.

10. Gambhir M, Gupta Sh (2015) Review of Turn around Point Long Period Fiber Gratings. Journal of Sensor Technology 5: 81-89.

11. Esfandyarpour R, Esfandyarpour H, Javanmard M, Harris JS, Davis RW, et al. (2014) Microneedle Biosensor: A Method for Direct Label-free Real Time Protein Detection. Sensors and Actuators B Chemistry 177: 848855.

12. Martinez Rios A, Monzon Hernandez D, Torres Gomez I, Salceda Delgado G (2012) Long Period Fibre Gratings. Centro de Investigaciones en Optica, Mexico.

13. Śmietana M, Bock WJ, Mikulic P, Ng A, Chinnappan R, et al. (2011) Detection of bacteria using bacteriophages as recognition elements immobilized on long-period fiber gratings. Optics Express 19(9): 79717978.

14. Korposh S, Chianella I, Guerreiro A, Caygill S, Piletsky S, et al. (2014) Selective vancomycin detection using optical fibre long period gratings functionalised with molecularly imprinted polymer nanoparticles. The Analyst 139(9): 2229-2236.

15. Zawisza R, Eftimov T, Mikulic P, Bock WJ, Jaroszewicz LR, et al. (2018) Ambient Refractive-Index Measurement with Simultaneous Temperature Monitoring Based on a Dual-Resonance Long-Period Grating Inside a Fiber Loop Mirror Structure. Sensors 18(7): 2370.

16. Brzozowska E, Koba M, Śmietana M, Górska S, Janik M, et al. (2016) Label-free Gram-negative bacteria detection using bacteriophageadhesin-coated long-period gratings. Biomedical Optics Express 7(3): 829-840.

17. Eakin RE, Snell EE, Williams RJ (1940) Egg-white injury in chicks and its relationship to a deficiency of vitamin $\mathrm{H}$ (biotin). Journal of Biological Chemistry 136: 801-802.

18. Kuramitz H, Sugawara K, Tanaka S (2000) Electrochemical Sensing of Avidin-Biotin Interaction Using Redox Markers. Electroanalysis 12: 1299-1303.

19. Zawisza R, Eftimov T, Mikulic P, Chinifooroshan Y, Celebańska A, et al. (2018) Dual-resonance long-period grating in fiber loop mirror structure for liquid refractive index measurement. Opto-electronics Review 26(1): 24-28.

20. Chiniforooshan Y, Celebańska A, Janik M, Mikulic P, Haddadb F, et al. (2017) Double resonance long period fiber grating for detection of $\mathrm{E}$. coli in trace concentration by choosing a proper bacteriophage. 25th International Conference on Optical Fiber Sensors.

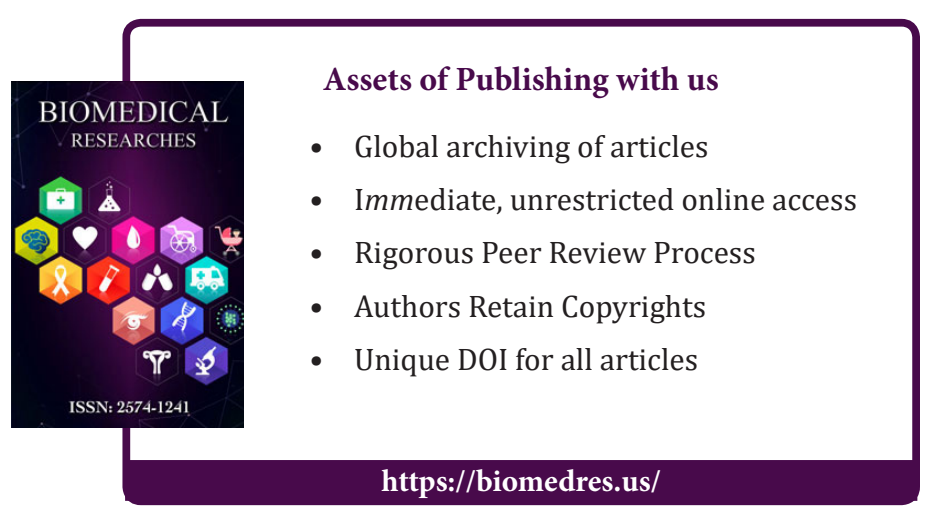

Cite this article: Renata Zawisza, Leszek R Jaroszewicz, Anna Celebańska, Predrag Mikulic, Wojtek J Bock. Dual-Resonance Long Period Grating in Fiber Loop Mirror Based Platform for Cheap Biomedical Sample Detection with High Resolution. Biomed J Sci\&Tech Res 10(3)2018. BJSTR. MS.ID.001967. DOI: 10.26717/ BJSTR.2018.10.001967. 